\title{
Leonurine protects cardiac function following acute myocardial infarction through anti-apoptosis by the PI3K/AKT/GSK3ß signaling pathway
}

\author{
LIN XU ${ }^{1-3}$, XUEJUN JIANG ${ }^{1-3}$, FANG WEI $^{1-3}$ and HONGLING ZHU ${ }^{1-3}$ \\ ${ }^{1}$ Department of Cardiology, Renmin Hospital of Wuhan University; ${ }^{2}$ Cardiovascular Research Institute; \\ ${ }^{3}$ Hubei Key Laboratory of Cardiology, Wuhan University, Wuhan, Hebei 430060, P.R. China
}

Received August 29, 2017; Accepted May 10, 2018

DOI: $10.3892 / \mathrm{mmr} .2018 .9084$

\begin{abstract}
Leonurine is a compound derived from Herba leonuri, which has been reported to protect cardiac tissue against ischemic injury via antioxidant and anti-apoptosis effects. The present study investigated whether these effects may be applied to acute myocardial infarction (MI) and examined the underlying mechanisms of leonurine treatment. A rat model of MI was induced by coronary artery ligation. Leonurine was administered at $15 \mathrm{mg} / \mathrm{kg} /$ day by oral gavage following the onset of MI. Rats in the sham group and the saline group were administered with an equal volume of saline. Echocardiography, Masson's trichrome staining, and terminal-deoxynucleotidyl transferase-mediated dUTP nick end labeling assays were performed 28 days post MI. The expression of B-cell lymphoma-2 and Bax were assessed by western blot analysis and reverse transcription-quantitative polymerase chain reaction. Phosphoinositide 3-kinase (PI3K), protein kinase $B$ and glycogen synthase kinase-3 $\beta$ (GSK3 $\beta$ ) protein expression were investigated by western blot analysis. Leonurine significantly alleviated collagen deposition and MI size, inhibited cell apoptosis and improved myocardial function. This was accompanied by significantly increased levels of phosphorylated (p)-PI3K, p-AKT, p-GSK3 $\beta$ and Bcl-2, as well as significantly decreased levels of caspase3, cleaved-caspase 3 and Bax following MI. The results demonstrated that leonurine exerts potent cardio-protective effects in a rat model
\end{abstract}

Correspondence to: Dr Hongling Zhu, Department of Cardiology, Renmin Hospital of Wuhan University, 238 Jiefang Road, Wuhan, Hebei 430060, P.R. China

E-mail: honglingzhu@whu.edu.cn

Abbreviations: PI3K, phosphoinositide 3-kinase; GSK3 $\beta$, glycogen synthase kinase- $3 \beta$

Key words: leonurine, myocardial infarction, anti-apoptosis, phosphoinositide 3-kinase, protein kinase $\mathrm{B}$, glycogen synthase kinase-3 $\beta$ of MI by inducing anti-apoptotic effects by activating the $\mathrm{PI} 3 \mathrm{~K} / \mathrm{AKT}$ /GSK $3 \beta$ signaling pathway.

\section{Introduction}

Ischemic heart disease, especially myocardial infarction (MI), remains the leading cause of death in the world (1). Sustained myocardial ischemia causes multiple kinds of damage to cardiac tissue (2). It is of great importance to alleviate detrimental damage by MI with pharmacological treatment.

Apoptosis plays a key role in the pathogenesis of myocardial ischemia, indicating that inhibition of myocardial apoptosis could decrease or alleviate the loss of terminally differentiated myocardial cells $(3,4)$. Thus, an anti-apoptosis and anti-necrotic strategy serves as a potential target for multiple therapies of myocardial ischemia (5). The phosphoinositide 3-kinase (PI3K)/AKT/glycogen synthase kinase-3 $\beta$ (GSK3 $\beta$ ) pathway plays a key role in the regulation of anti-apoptosis in multiple tissues, including heart, brain, ovaries and kidney.

Herba leonuri, one kind of traditional Chinese medicine, has been widely used to treat dysmenorrhea, menoxenia and gynecological dysfunctions (6). Several reports of pharmaceutical preparations based on $\mathrm{H}$. leonuri suggest that it may have protective effects against cardio-cerebral vascular diseases $(7,8)$. Leonurine, a specific component of $\mathrm{H}$. leonuri, has been reported to reveal cardio-protective effects in vivo and in vitro $(9,10)$. Leonurine's effects also result in increased phosphorylation of AKT and expression of HIF-1, Surviving and VEGF in rats with myocardial ischemia. Leonurine also inhibited apoptosis in $\mathrm{H} 9 \mathrm{c} 2$ cell under hypoxia by increasing expression of the anti-apoptotic protein Bcl-2 and decreasing expression of the pro-apoptotic protein Bax (11). Although multiple studies have been carried out to study the effect of Leonurine on myocardial protection, the underlying mechanisms remain unclear. Thus, in the present study, we investigated the mechanism of cardio-protection by Leonurine in rats with $\mathrm{MI}$.

\section{Materials and methods}

Our study included 45 male SD rats (200-250 g) from Hunan Provincial Center for Disease Control and Prevention in 
China. They were cared for under a room temperature of 22-25 ${ }^{\circ} \mathrm{C}$ and 12:12-h light/dark cycle environment. Five rats were housed in each cage with sufficient food and water. We designed experimental procedures and protocols conformed to the Guide for the Care and Use of Laboratory Animals from the US National Institutes of Health and to the Institutional Animal Care Committee from Wuhan University in China (wdrm-20140812).

Model of MI and in vivo leonurine administration. A total of 45 rats were randomly divided into three groups: i) sham-operated group (Sham); ii) MI with saline group (NS); and iii) MI with Leonurine group (Leonurine). The model of MI was operated as previous study, male SD rats were anesthetized intra-peritoneally with $3 \%$ pentobarbital sodium $(30 \mathrm{mg} / \mathrm{kg})$, then intubated and ventilated at a respiratory rate of $70 \mathrm{times} / \mathrm{min}$. A thoracotomy was implemented from the third intercostal space on the left side of rats. After the epicardium was stripped, the left coronary artery was ligated $2 \mathrm{~mm}$ below the left atrial appendage with 6-to-0 polypropylene. The electrocardiogram (ECG) of rats was recorded by ECG monitoring, and a successful MI model was determined by ST-segment elevation in Leads II and III in ECG and pallor appearance in the infarcted area. The rats in sham group underwent thoracotomy without left anterior descending (LAD) ligation and served as control.

The rats in the Leonurine group were treated with Leonurine (24697-74-3; Shanghai Yanyi Biotechnology Co., Ltd., Shanghai, China) at $15 \mathrm{mg} / \mathrm{kg} / \mathrm{d}$ by oral gavage after the onset of MI for 28 days. The rats in sham group and NS group were administered with equal volume of saline in the same manner. According to previous study, Leonurine at both 15 and $30 \mathrm{mg} / \mathrm{kg}$ decreased myocardial fibrosis and down regulated Nox4-ROS-NF- $\kappa$ B pathway, while there was no significant difference in inhibiting cell fibrosis between these two doses (12). And in Liu et al study, only $15 \mathrm{mg} / \mathrm{kg} /$ day of Leonurine significantly reduced neurological deficits and brain infarct volume compared with $7.5 \mathrm{mg} / \mathrm{kg}$ /day of leonurine or vehicle group (13). Therefore the dose of leonurine in our present study is relatively low but safe and effective without causing any side effects.

Echocardiography. On Day 1 and Day 28 after the MI surgery, all the rats were anesthetized with their chest shaved. We used a transthoracic echocardiography (Acuson, Mountain View, CA, USA) which was equipped with a 3- to 7-MHz probe to investigate their left ventricular (LV) dimension and heart function in the long-axis and short-axis views in a blind manner. The animals with visual infarct areas less than $20 \%$ in the short-axis view were excluded in order to minimize the variation in infarct areas. LV end-systolic diameter (LVESD), LV end-diastolic diameter (LVEDD), and ejection fractional (EF) were assessed.

Histopathological examinations. All the rats were sacrificed using a pentobarbital overdose $(200 \mathrm{mg} / \mathrm{kg})$ after the echocardiography assessment. The heart was immediately removed into diastole with $10 \% \mathrm{KCl}$ and the ventricles parts were fixed in $10 \%$ ( $\mathrm{vol} / \mathrm{vol}$ ) buffered formalin solution. Masson's trichrome stain and immunohistochemistry were conducted on five different transversal levels in the peri-injection site subsequently after embedded. In all 3 animal groups, MI area and collagen content were investigated in sections obtained from the border zone (about $2 \mathrm{~mm}$ from the infarction zone edge) via light microscopy. In each LV transverse section, 4 random microscopic fields were chosen in a blind fashion and subsequently examined. By computer image analysis software, the surface area of infarct wall and the entire surface area of the left ventricle (magnification, x10) was automatically calculated, and the ratio of the former to the latter was identified as the Infarct size $(\%)$. The image analysis software was also used to calculate the Collagen content (\%), which was identified as the ratio of the area of collagen to the area of the entire high-power field (magnification, x200).

TUNEL assay. Twenty-eight days post the MI surgery, the apoptotic cells in the LV were examined using a TUNEL assay (Roche Diagnostics GmbH, Mannheim, Germany). As instructed by the manufacturer, we stained the nuclear DNA fragments in the apoptotic cells using the transferase dUTP nick-end labeling technique and the hematoxylin counterstaining. In a blind manner, we selected three random fields (magnification, $x 400$ ) in each slide and calculated the TUNEL-positive nuclei via a microscopy. The apoptotic index (\%) was termed as the ratio of the number of TUNEL-positive nucleolus to the total number of nuclei.

Reverse transcription-quantitative polymerase chain reaction $(R T-q P C R)$. At 28 days post the MI surgery, we collected the peri-infarct area of the hearts to do the total RNA isolation with TRIzol reagent (Invitrogen; Thermo Fisher Scientific, Inc., Waltham, MA, USA). According to the manufacturer's descriptions, we reverse transcribed complementary DNA (cDNA) from total RNA with oligo (dT) primers (Shanghai Sangon BET Company, Shanghai, China), then amplified DNA fragments with a SYBR-Green-based assay kit (Invitrogen; Thermo Fisher Scientific, Inc.). The RT-qPCR started at $42^{\circ} \mathrm{C}$ for $1 \mathrm{~h}, 95^{\circ} \mathrm{C}$ for $5 \mathrm{~min}$ to make the cDNA; then followed the condition including 1 cycle at $50^{\circ} \mathrm{C}$ for $2 \mathrm{~min}$ and $95^{\circ} \mathrm{C}$ for $10 \mathrm{~min}, 40$ cycles at $95^{\circ} \mathrm{C}$ for $30 \mathrm{sec}$ and $60^{\circ} \mathrm{C}$ for $30 \mathrm{sec}$. The RNA level of Bax and Bcl-2 were calculated according to CT values between the experimental group and the control group. $\beta$-actin was used as the housekeeping gene. The specific primer sequence and amplicon size of the selected genes are listed in Table I.

Western blot analysis. At 28 days post the MI surgery, western blotting was conducted with the peri-infarct area of the hearts. The Bicinchoninic Acid protein assay (Beyotime Institute of Biotechnology, Haimen, China) was used to examine the protein concentration. We added $50 \mu \mathrm{g}$ of denatured protein in each hole of the $10 \%$ SDS-polyacrylamide gels and transferred the protein to a PVDF membrane (EMD Millipore, Billerica, MA, USA). Membranes were probed with first antibodies against PI3K (1:1,000; Cell Signaling Technology, Inc., Danvers, MA, USA), p-PI3K (1:300; Santa Cruz Biotechnology, Inc., Dallas, TX, USA), AKT (1:1,000; Cell Signaling Technology, Inc.), p-AKT (1:800; Cell Signaling Technology, Inc.), GSK3 $\beta$ (1:1,000; Abcam, 
Table I. Specific primer sequence and amplicon size of the selected genes rat $\beta$-actin, Bax and BCLcl-2.

\begin{tabular}{|c|c|c|c|}
\hline Name & Primer & Sequence & Size \\
\hline \multirow[t]{2}{*}{ Rat $\beta$-actin } & $\mathrm{F}$ & 5'-CACGATGGAGGGGCCGGACTCATC-3' & $240 \mathrm{bp}$ \\
\hline & $\mathrm{R}$ & 5'-TAAAGACCTCTATGCCAACACAGT-3' & \\
\hline \multirow[t]{2}{*}{ Rat Bax } & $\mathrm{F}$ & 5'-CAGGCGAATTGGCGATGAAC-3' & $134 \mathrm{bp}$ \\
\hline & $\mathrm{R}$ & 5'-CCCAGTTGAAGTTGCCGTCT-3' & \\
\hline \multirow[t]{2}{*}{ Rat BCLcl-2 } & $\mathrm{F}$ & 5'-CTGGCATCTTCTCCTTCCAG-3' & $181 \mathrm{bp}$ \\
\hline & $\mathrm{R}$ & 5'-CGGTAGCGACGAGAGAAGTC-3' & \\
\hline
\end{tabular}

Bcl-2, B-cell lymphoma-2; bp, base pairs; F, forward; R, reverse.
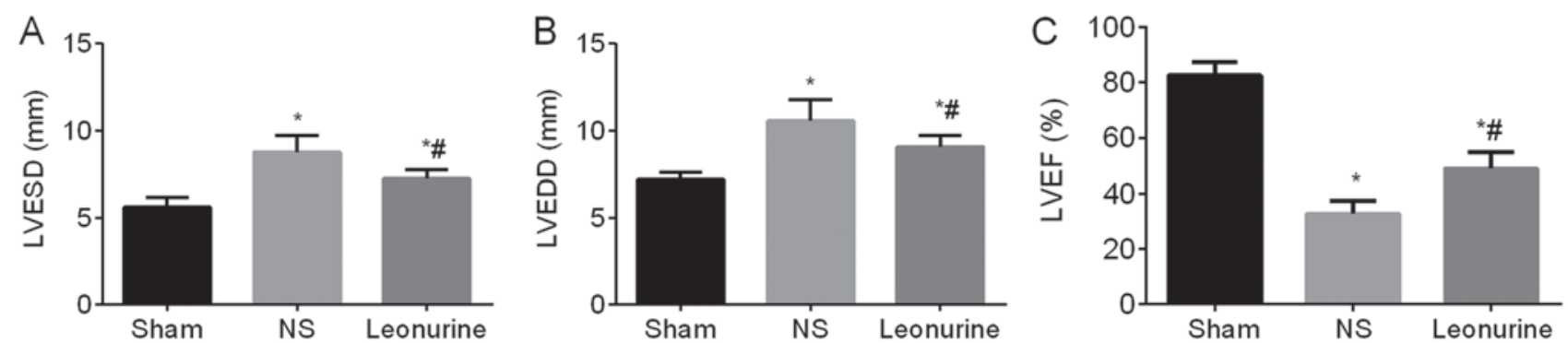

Figure 1. Echocardiographic evaluation of left ventricular function, including (A) LVEDD, (B) LVESD and (C) LVEF 28 days after myocardial infarction. The data are expressed as the mean \pm standard deviation. " $\mathrm{P}<0.05$ vs. sham, ${ }^{~} \mathrm{P}<0.05$ vs. NS. $\mathrm{n}=12$ in each group. LVESD, left ventricular end-systolic diameter; LVEDD, left ventricular end-diastolic diameter; LVEF, left ventricular ejection fractional; NS, saline group.

Cambridge, UK), p-GSk3 3 (1:800, Abcam), Caspase3 (1:300; Santa Cruz Biotechnology, Inc.), Cleaved-caspase3 (1:1,000; Cell Signaling Technology, Inc.), Bax (1:200; Santa Cruz Biotechnology, Inc.), Bcl-2 (1:200; Santa Cruz Biotechnology, Inc.), and second antibodies as HRP goat-anti-rat, HRP goat-anti-rabbit, HRP rabbit-anti-goat. Finally the blots were analyzed with the ImageJ software after been visualized by the chemiluminescence methods. GAPDH was termed as an internal control.

Statistical analysis. The data were showed as the mean \pm standard deviation. We used one-way ANOVA's test and the Student-Newman-Keuls' test to analyze the data. $\mathrm{P}<0.05$ was considered to indicate a statistically significant difference.

\section{Results}

Totally 45 rats were initially used in this project. According to original weight from low to high, 45 rats were ear-marked with tags ranking number 1-45. Then, we transcribed random numbers from the random number table from the first number at the first row and copied down 45 numbers between 1 and 99. Each number was divided by 3, and the remainder 1, 2 and 3 is on behalf of the group sham, NS and Leonurine respectively. In case one or more groups had more than 15 rats, for example the NS group had 16 rats, we continued to record 1 number between 1 and 99 from the 46 number and divided by 3 , then this remainder stood for the corresponding rat would be included into next group which was less than 15 rats. Finally, the above three groups owned
15 rats, respectively. A total of 3 rats (in sham group) were dead because of hyper-anesthesia before surgery. A total of 5 rats (3 in NS group and 2 in Leonurine group) died because of left heart failure combined with wound infection within 1 week post MI experiments. One rat (in Leonurine group) was excluded as infarct size was less than $20 \%$. None of the rest rats died with unexplained reasons or suffered from any side effects such as vomiting, decreased body weight, hair loss or physical weakness. At 28 days after the MI surgery, all the rats were investigated on the heart functions with Echocardiography before been sacrificed. And for each group, we as well chose transcribed random numbers from the random number table from the first number at different rows and copied down 12 numbers between 1 and 99, and divided by 2 , with the remainder 1 and 2 on behalf of histological staining (6 rats) and biochemical testing (6 rats). Thus 6 rats from each group were chosen from random selection and sacrificed to measure infarct size, collagen content and apoptosis index, while another 6 rats from each group were sacrificed to perform western blot and RT-PCR analyses.

$L V$ diameter and LVEF. The first day after MI, the NS and the Leonurine group showed an obvious impaired heart function (decreased LVEF and increased LV diameters) compared with the sham group, while the NS group and the Leonurine group showed similar heart function between each other (data not shown). At 28 days after MI, the MI groups still showed an impaired heart function (significant increase in the LVESD and LVEDD and an obvious decrease in the LVEF) compared to the sham group (LVESD, 5.58 $\pm 0.15 \mathrm{~mm}$; LVEDD, 
A

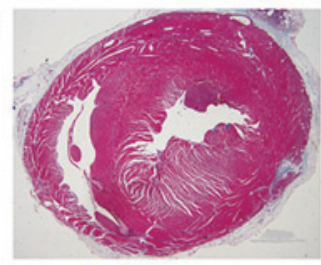

Sham

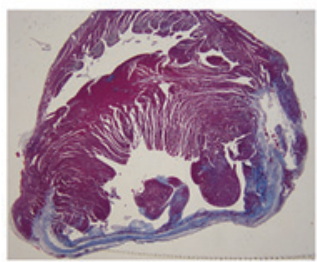

NS

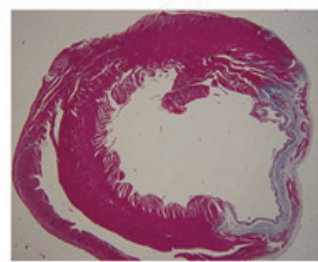

Leonurine

B

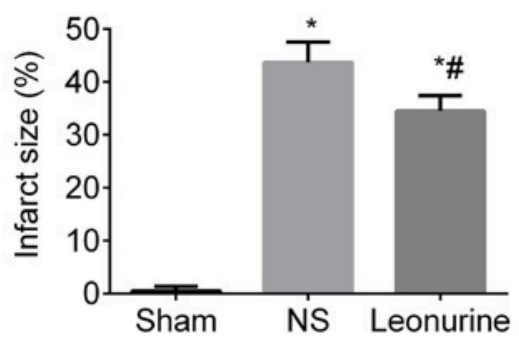

Figure 2. Infarct size 28 days after myocardial infarction. (A) Representative pictures of left ventricles from each group after Masson's Trichrome staining (magnification, x10). (B) Infarct size as percentages at 28 days. Infarct size is calculated from the ratio of surface area of infarct wall and the entire surface area of the left ventricle. The data are expressed as the mean \pm standard deviation. ${ }^{*} \mathrm{P}<0.05$ vs. sham, ${ }^{\text {}} \mathrm{P}<0.05$ vs. NS. $\mathrm{n}=6$ in each group. NS, saline group.

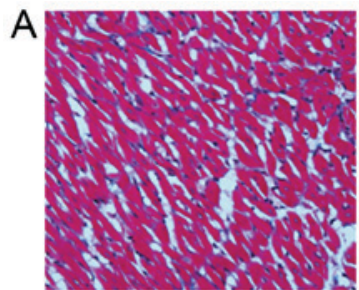

Sham

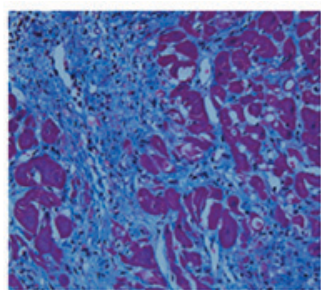

NS

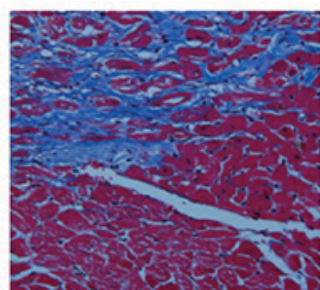

Leonurine

B

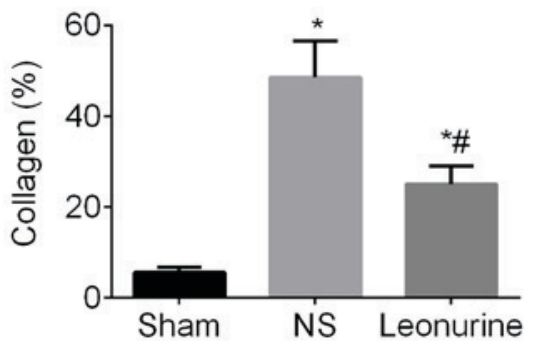

Figure 3. Collagen content 28 days after myocardial infarction. (A) Representative pictures of left ventricles from each group after Masson's Trichrome staining (magnification, x200). (B) Collagen content as percentages at 28 days. Collagen content is calculated from the ratio of the collagen area to the area of the entire high-power field. The data are expressed as the mean \pm standard deviation. ${ }^{*} \mathrm{P}<0.05$ vs. sham, ${ }^{~} \mathrm{P}<0.05$ vs. NS. $\mathrm{n}=6$ in each group. NS, saline group.

$7.17 \pm 0.13 \mathrm{~mm}$; LVEF, 82.62 $\pm 1.41 \%)$. However, the Leonurine group (LVESD, 7.15 $\pm 0.16 \mathrm{~mm}$; LVEDD, 9.07 $\pm 0.18 \mathrm{~mm}$; LVEF, $48.93 \pm 1.73 \%$ ) showed not only a remarkable reduction in $\mathrm{LV}$ diameters $(\mathrm{P}<0.05$; Fig. $1 \mathrm{~A}$ and $\mathrm{B})$ but also an increase in LVEF $(\mathrm{P}<0.05$; Fig. 1C) compared with the NS group (LVESD, 8.73 $\pm 0.27 \mathrm{~mm}$; LVEDD, $10.53 \pm 0.36 \mathrm{~mm}$; LVEF, $32.73 \pm 1.38 \%)$.

Infarct size. At 28 days after MI, the Leonurine group $(34.49 \pm 1.19 \%)$ showed a significant reduction in infarct size compared with the NS $(43.66 \pm 1.60 \%)$ group $(\mathrm{P}<0.05$; Fig. 2$)$.

Collagen content. At 28 days after MI, collagen content was remarkably increased $(\mathrm{P}<0.05$; Fig. 3$)$ in the MI group compared with the sham $(5.52 \pm 0.46 \%)$ group. After Leonurine $(24.99 \pm 1.63 \%)$ treatment, the collagen content was remarkably decreased compared with the results of the NS group $(48.55 \pm 3.24 \%)$.

Apoptosis. At 28 days after MI, the NS $(27.21 \pm 0.84 \%)$ and Leonurine group $(17.28 \pm 0.82 \%)$ exhibited an obvious increase $(\mathrm{P}<0.05$; Fig. 4$)$ of the apoptosis index compared with the sham group $(2.5 \pm 0.66 \%)$, while the Leonurine group showed a smaller apoptosis index $(\mathrm{P}<0.05 ;$ Fig. 4) compared with the NS group (Fig. 4).

Expression of PI3K/AKT/GSK3 $\beta$ signaling pathway and apoptosis-related proteins. The gene expression of Bax in NS group 28 days after MI is higher than the expression in the sham group, though not to a statistically significant extent (Fig. 5). However, the Leonurine group had significantly decreased the gene expression of Bax while increased the 


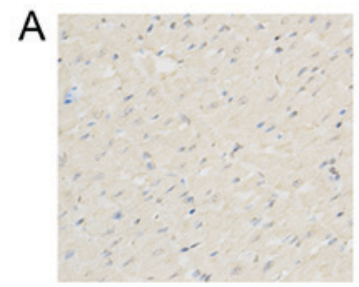

Sham

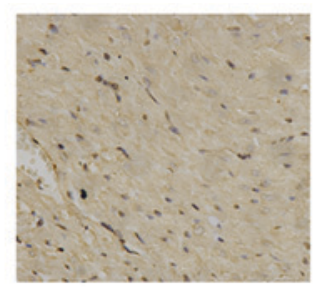

NS

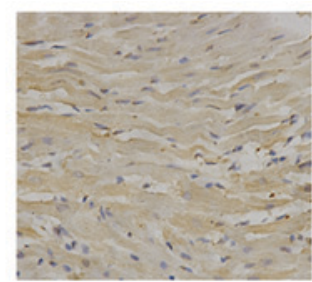

Leonurine

B

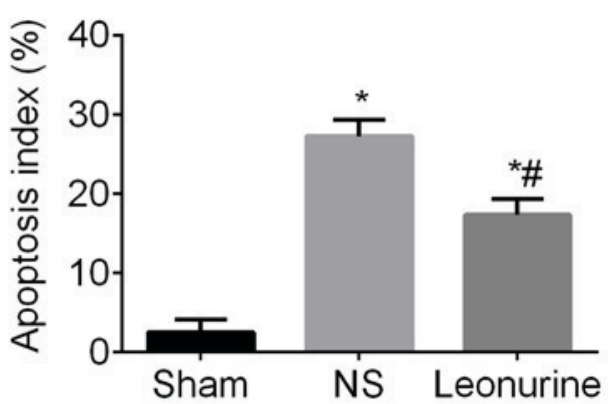

Figure 4. Cardiomyocyte apoptosis 28 days after myocardial infarction. (A) Immunohistochemistry of TUNEL staining in the border zone (Magnifiaction, $\mathrm{x} 200$ ). (B) The apoptotic index was quantified at 28 days. The data are expressed as the mean \pm standard deviation. ${ }^{*} \mathrm{P}<0.05$ vs. sham, ${ }^{\#} \mathrm{P}<0.05$ vs. NS; $\mathrm{n}=6$ in each group. NS, saline group.

A

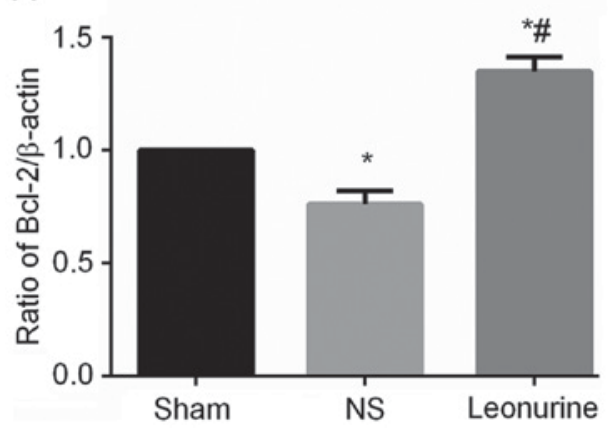

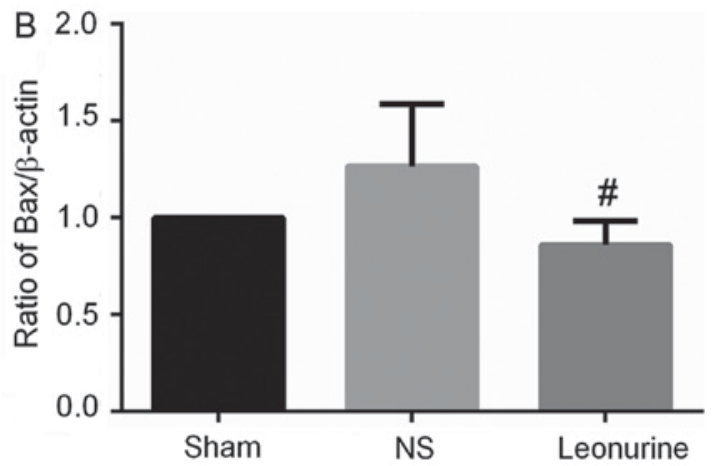

Figure 5. The level of Bcl-2 and Bax mRNA 28 days after MI. (A) The level of Bcl-2 and (B) Bax mRNA 28 days after MI. The data are expressed as the mean \pm standard deviation. ${ }^{*} \mathrm{P}<0.05$ vs. sham, ${ }^{\#} \mathrm{P}<0.05$ vs. NS; $\mathrm{n}=6$ in each group. NS, saline group; Bcl-2, B-cell lymphoma 2 ; MI, myocardial infarction.

gene expression of Bcl-2 compared with the result in the NS group (Fig. 5A). Thus, the ratio of Bcl-2/Bax was significantly increased in the Leonurine group compared with that of NS group. The NS group showed a smaller ratio of Bcl-2/Bax than the sham group. The protein expression of $\mathrm{Bax}$ and $\mathrm{Bcl}-2$ had the same development trend in genetic levels (Fig. 6A).

The protein level of PI3K in all the groups had no significant difference, but MI significantly decreased the level of p-PI3K. Treatment with Leonurine significantly up-regulated the phosphorylation of PI3K, accompanied with a significantly increased ratio of p-PI3K/PI3K (Fig. 6B).

The protein level of AKT was significantly decreased in the Leonurine group compared with the NS and sham group. The protein level of p-AKT was decreased significantly in the NS group compared with the sham group. After treatment with Leonurine, p-AKT was significantly up-regulated, so as to significantly increase the ratio of $\mathrm{p}-\mathrm{AKT} / \mathrm{AKT}$ in the Leonurine group (Fig. 6C).

The total protein level of GSK $3 \beta$ in the three groups had no obvious difference. However the p-GSK3 $\beta$ was significantly down-regulated in the NS group and significantly up-regulated in response to Leonurine treatment. Moreover, the ratio of p-GSK3 $\beta /$ GSK $3 \beta$ was significantly increased in the Leonurine group compared to that of the NS group (Fig. 6D).

Caspase 3 was significantly decreased in the NS group and Leonurine group compared with the sham group. The cleaved-caspase 3 had been activated and cleaved in the NS group. However treatment with Leonurine significantly reduced the cleaved-caspase 3 expression. Thus the ratio of cleaved-caspase $3 /$ caspase 3 is significantly decreased in Leonurine group compared with that in NS group and sham group (Fig. 6E).

\section{Discussion}

The present study demonstrated that Leonurine significantly decreased the stretch of the infarct zone, alleviated the deposition of collagen, inhibited the apoptosis of cardiomyocytes, prevented LV dilation and improved cardiac function. These effects were due to that the Leonurine activated PI3K/AKT/GSK $3 \beta$ signaling pathway and decreased the expression of pro-apoptosis protein Bax and the activation of 
A a
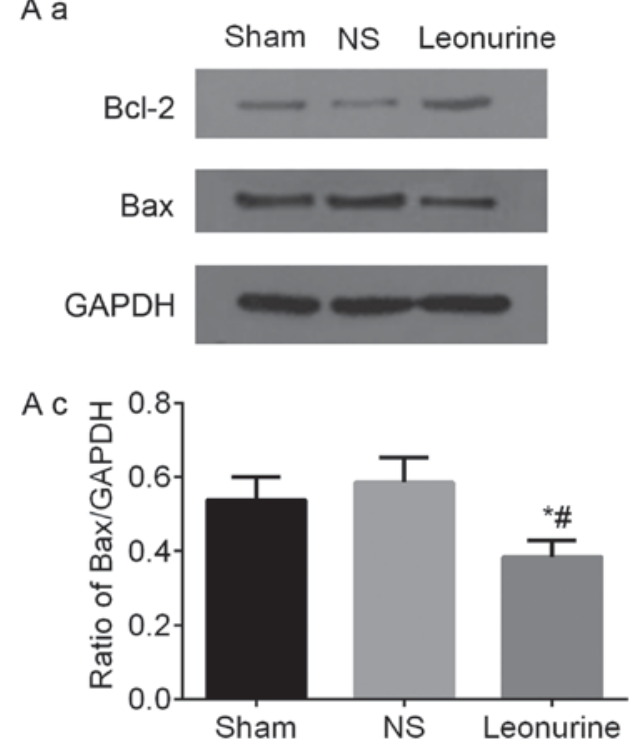

B a
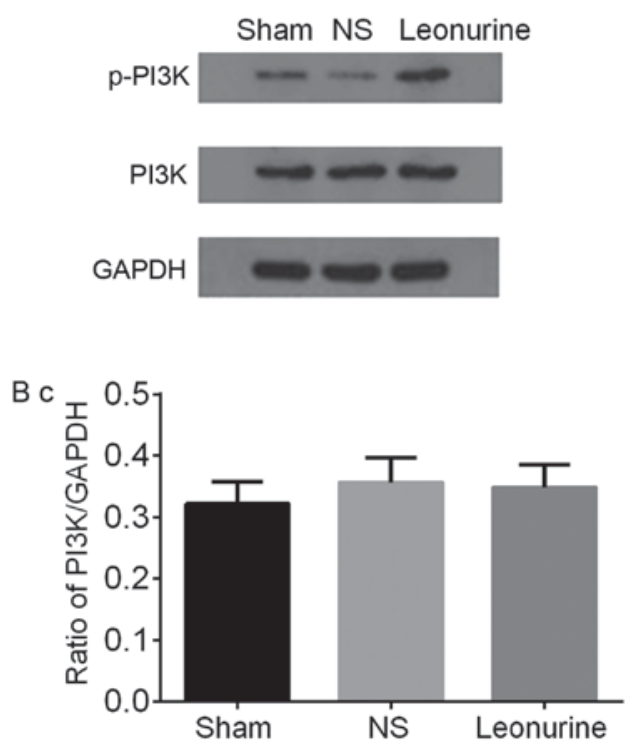

$\mathrm{Ca}$

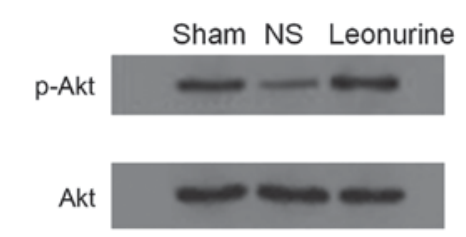

GAPDH

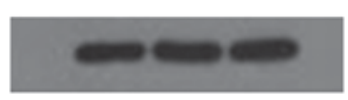

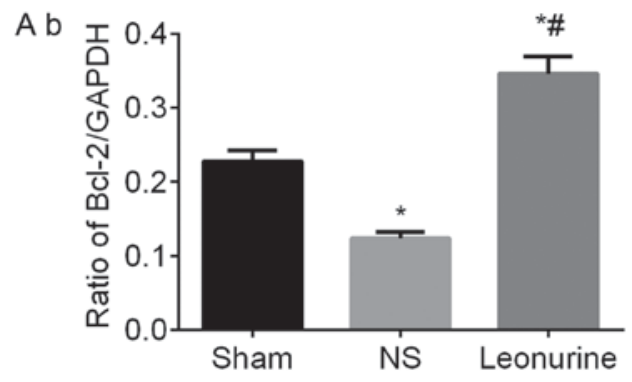

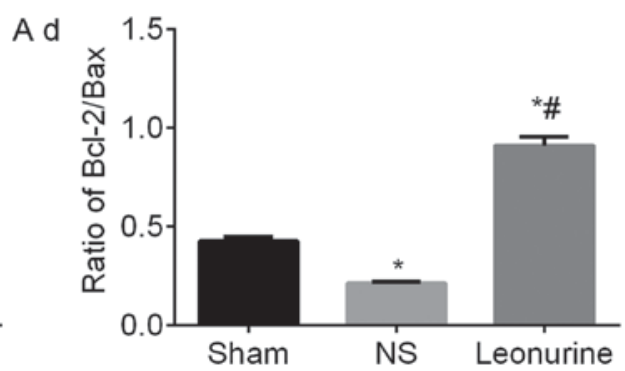

$\mathrm{Bb}$
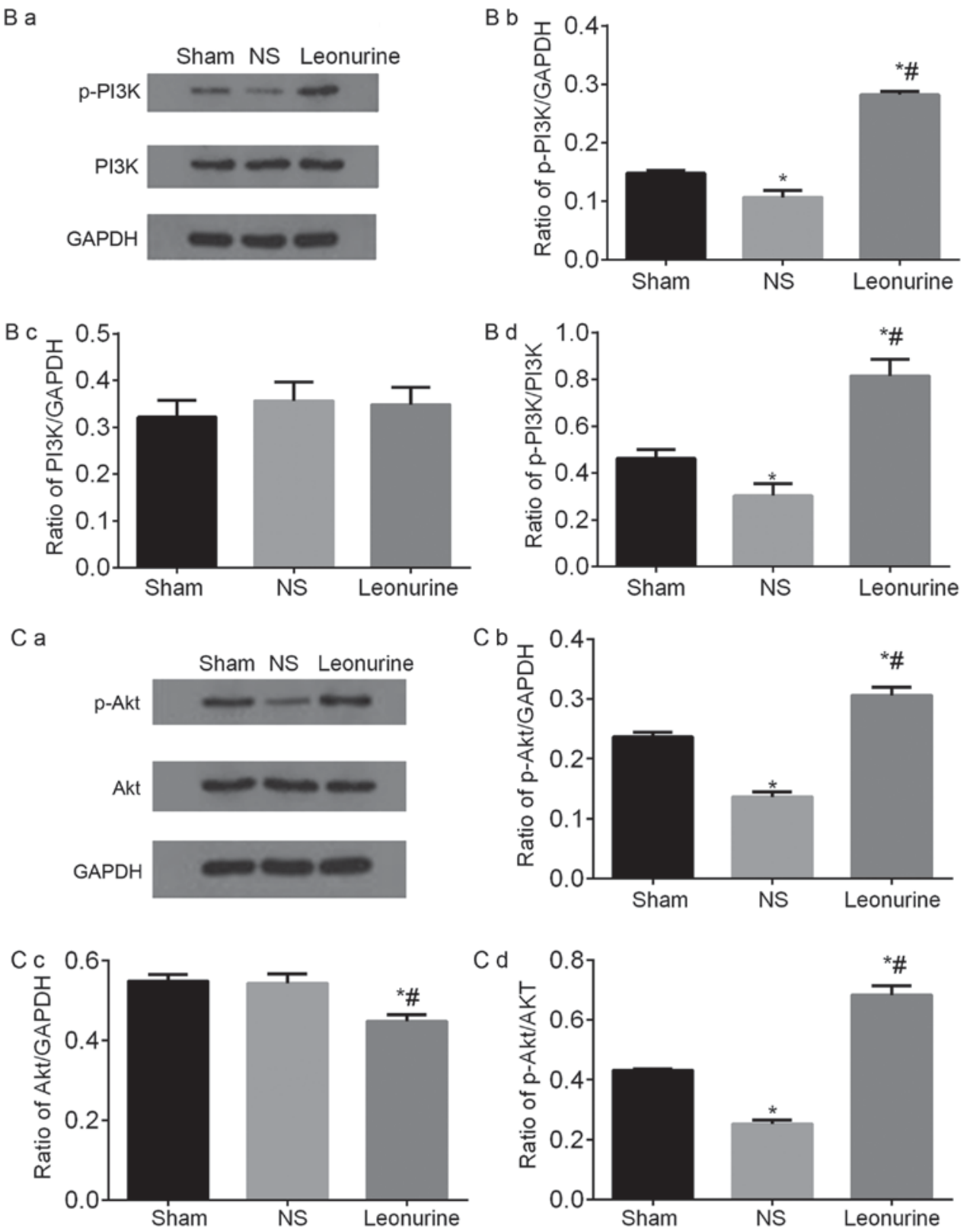

$C d$

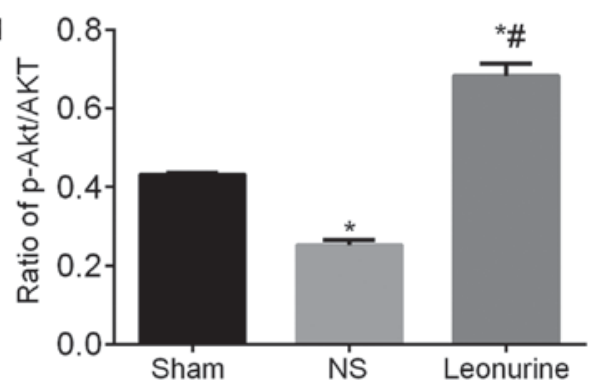

Figure 6. Representative immunoblots and quantified results of (A) Bcl-2 and Bax, (B) p-PI3K and PI3K, (C) p-AKT and AKT proteins by western blot analysis 28 days after myocardial infarction. The data are expressed as the mean \pm standard deviation. ${ }^{*} \mathrm{P}<0.05$ vs. sham, ${ }^{\#} \mathrm{P}<0.05$ vs. NS. $\mathrm{n}=6$ in each group. 
$\mathrm{D}$ a
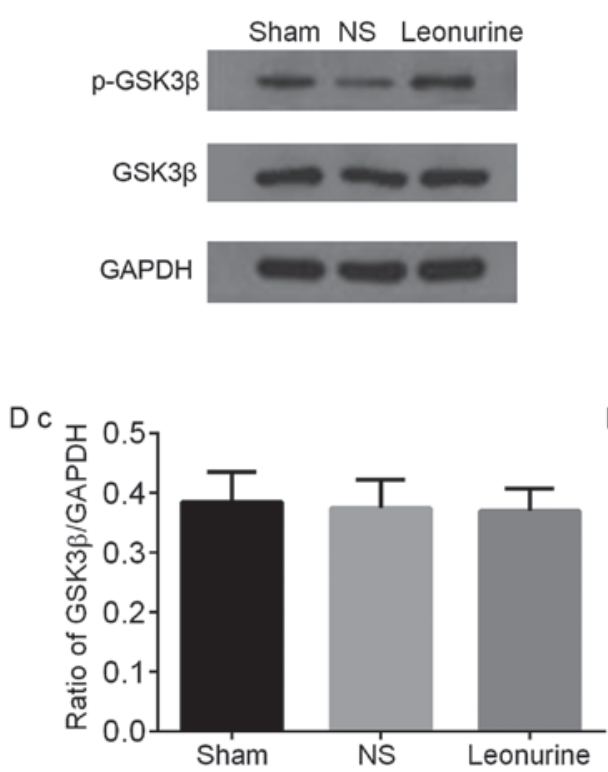

E a
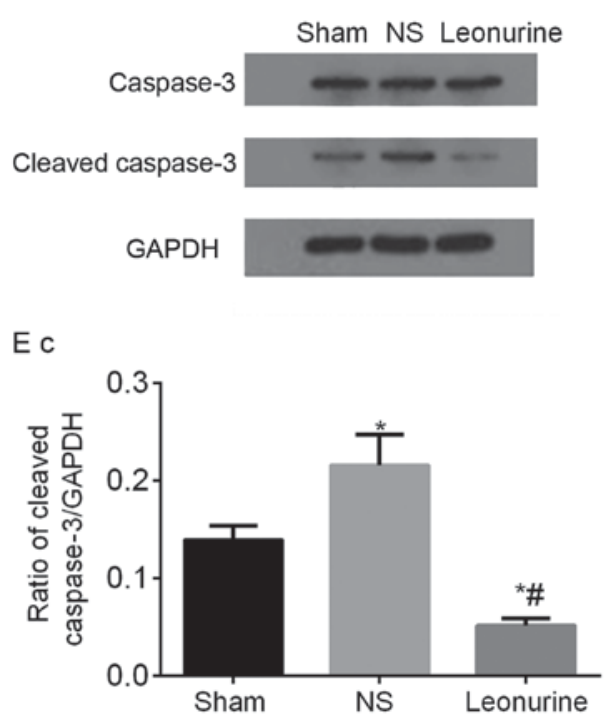

$\mathrm{Db}$

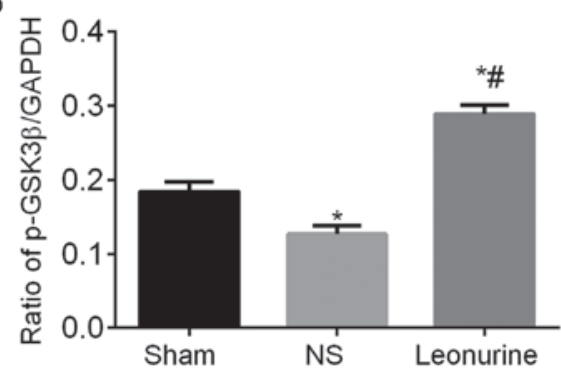

$\mathrm{Dd}$
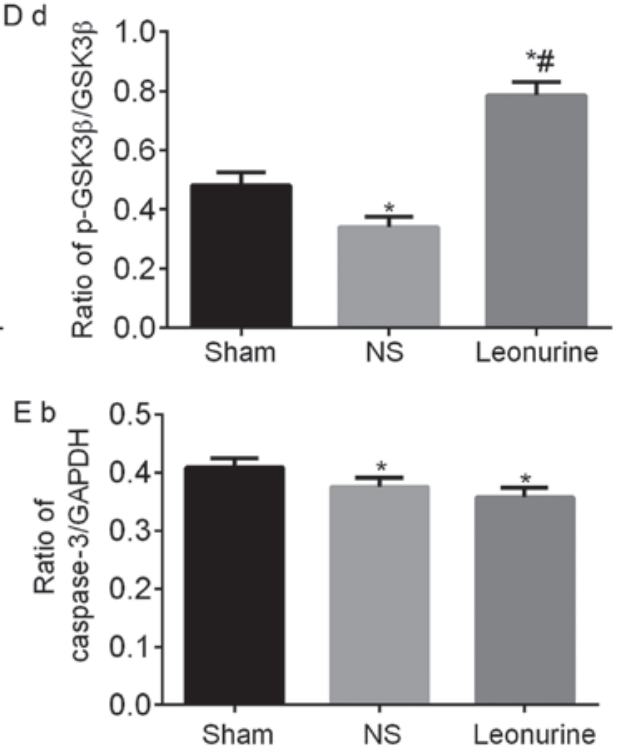

Ed

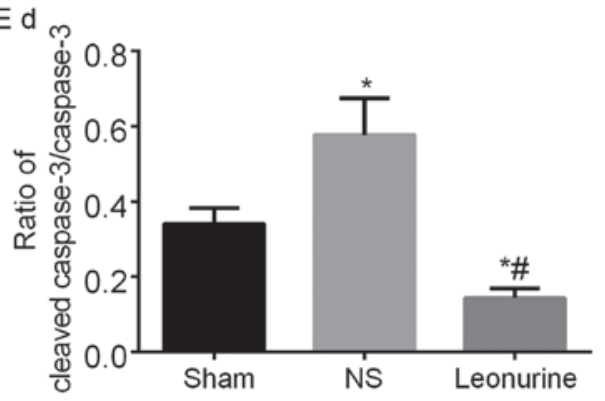

Figure 6. Continued. Representative immunoblots and quantified results of (D) p-GSK3 $\beta$ and GSK3 $\beta$ and (E) Caspase3 and Cleaved-caspase3 proteins by western blot analysis 28 days after myocardial infarction. The data are expressed as the mean \pm standard deviation. ${ }^{*} \mathrm{P}<0.05$ vs. sham, ${ }^{*} \mathrm{P}<0.05 \mathrm{vs}$. NS. $\mathrm{n}=6$ in each group. PI3K, phosphoinositide 3-kinase; GSK3 $\beta$, glycogen synthase kinase-3 $\beta$.

apoptosis marker caspase 3 , and increased the expression of anti-apoptosis protein $\mathrm{Bcl}-2$.

With the feature that myocardium is terminally differentiated tissue, it is critical to preserve cardiomyocyte viability against ischemia diseases. The wide occurrences of cell apoptosis in the ischemic heart, especially in the peri-infarct size, largely hinder the recovery of cardiac function. Therefore, activation of the anti-apoptosis signaling pathway and expression of specialized anti-apoptotic proteins is crucial in preserving cardiac function during ischemia.

The PI3K/AKT/GSK3 $\beta$ signaling pathway has been well documented in mediating growth, differentiation, proliferation and cell survival. In Yu-Shengyou' study, Dex induced anti-apoptotic effects through activating PI3K/AKT and the downstream target GSK3 $\beta$ against PAN-induced apoptosis in cultured podocytes (14).
PI3K, named phosphatidylinositol 3-kinase, specifically catalyzes the phosphorylation of the hydroxyl group on the 3 position of phosphatidylinositol (PI), forming 3 , 4-diphosphate phosphatidylinositol and 3, 4, 5-triphosphate phosphatidylinositol which are downstream messengers to activate AKT (15). AKT is a member of serine/threonine-protein kinases that contain SH2 (Src homology 2-like) domains and is commonly referred to as PKB.

$\mathrm{PI} 3 \mathrm{~K} / \mathrm{AKT}$ is a critical signaling pathway mediating survival, growth and apoptosis (16). In Hirokazu Ohashi' study, $\mathrm{PI} 3 \mathrm{~K} / \mathrm{AKT}$ induces the expression of surviving and decreases the activity of Caspase-3 to inhibit the apoptotic effects induced by Angiotensin II in microvascular endothelial cells (17). Moreover, EPO reduced myocardial apoptosis and enhanced cardiac-protection under hypoxia and ischemia conditions by activating the PI3K/AKT signaling pathway $(18,19)$. It is likely 
that in the Yizhun Zhu' study, PI3K/AKT plays an important role in ischemia heart diseases $(2,11)$.

GSK3 belongs to the serine/threonine-protein kinase family and consists of GSK $3 \alpha$ and GSK3 $\beta(20,21)$. GSK3 $\beta$, a main downstream substrate of AKT, participates in the $\mathrm{PI} 3 \mathrm{~K} / \mathrm{AKT}$ pathway regulating glycogen and protein synthesis, cell growth and anti-apoptosis $(22,23)$. Activated AKT can inhibit activation of GSK3 $\beta$ by phosphorylating GSK3 $\beta$ at the Ser residue, and decrease the expression of Caspase 3 as they induce anti-apoptosis effects $(24,25)$. AKT further facilitates the phosphorylation of GSK $3 \beta$ at the Ser residue and p-GSK3 $\beta$ induces $\beta$-catenin phosphorylation to promote cell survival (26). Wagner $C$ found that post-treatment rats model in vivo would obviously decrease the infarct size through mediating PI3K/AKT to increase the expression of $\mathrm{p}-\mathrm{GSk} 3 \beta$ (Ser9) (27).

In this study, we observed a small decrease of PI3K with a significant increase of $\mathrm{p}-\mathrm{PI} 3 \mathrm{~K}$ in the Leonurine group compared to the sham and NS group. Thus there was a significant increase of the ratio of $\mathrm{p}$-PI3K/PI3K after the treatment with Leonurine for 28 days after MI.

To test whether AKT expression relevantly changed, we investigated the level of AKT and $\mathrm{p}-\mathrm{AKT}$ in the three groups. We found that both $\mathrm{p}-\mathrm{AKT}$ and the ratio of $\mathrm{p}-\mathrm{AKT} / \mathrm{AKT}$ were significantly increased in the Leonurine group compared to the sham and NS group. We also observed a similar change in $\mathrm{p}-\mathrm{GSK} 3 \beta$. There was increased $\mathrm{p}-\mathrm{GSK} 3 \beta$ expression and a higher ratio of $\mathrm{p}-\mathrm{GSK} 3 \beta / \mathrm{GSK} 3 \beta$ in the Leonurine group compared to the sham and NS group. GSK $3 \beta$ further activated the Bax family and Caspase family of apoptosis mediating kinases $(26,28)$.

Thus, we suggest that treatment with Leonurine induces phosphorylation of PI3K, then the activated p-PI3K facilitates downstream molecule AKT to be phosphorylated. Subsequently, the p-AKT inhibits its downstream target protein GSK $3 \beta$. Ultimately the activated PI3K/AKT/GSK3 $\beta$ signaling axis induces anti-apoptotic effect with the treatment of Leonurine in rats 28 days after MI.

The Bcl-2 family is a potent regulator of apoptosis pathways and it includes both anti-apoptotic proteins and pro-apoptotic proteins. The ratio of Bcl-2 and Bax has been used as a marker representing the apoptosis effect. In the present study, we investigated the expression of Bcl-2 and Bax in both RNA level and protein level. We found that Bcl-2/Bax at both levels in the Leonurine group were significantly increased compared with that in the NS group. This indicates Leonurine induced anti-apoptosis effects by increasing the anti-apoptosis protein $\mathrm{Bcl}-2$ and decreasing the pro-apoptosis protein Bax.

Besides, caspase 3 is a potential member of caspase kinase family mediating cell apoptosis. Cleaved-caspase 3 is able to be induced by Bax and leads to apoptosis. As a result, it serves as a marker for the extent of pro-apoptosis. In our study we also exhibited that MI surgery caused significant apoptosis effect in NS group with decreased caspase 3 and largely increased cleaved-caspase 3 expression. However treatment with Leonurine was able to reverse this phenomenon and significantly decreased the expression of the activated form of caspase 3 and decreased the ration of cleaved caspase $3 /$ caspsase 3 at the protein level. This phenomenon was useful to alleviate the activation of the apoptosis pathway and provide protective effects to myocardial myocytes.

Thus decreased infarct size and improved cardiac function of rats are largely due to the activation of $\mathrm{PI} 3 \mathrm{~K} / \mathrm{AKT} / \mathrm{GSK} 3 \beta$ signaling pathway and subsequent up-regulation of anti-apoptosis proteins $\mathrm{Bcl}-2$, and down-regulation of pro-apoptosis protein Bax and cleaved-caspase 3 caused by treatment of Leonurine. Besides, several reports exhibited that Leonurine shows the effect of anti-oxidation (29), anti-inflammation (30), anti-myocardial fibrotic response (12) and enhancing mitochondrial function (31), which probably participate in the cardio-protection in our study as well.

A limitation of the present study was that no inhibitor of PI3K/AKT/GSK $3 \beta$ was set up to further validate the signaling pathway mechanism because of insufficient Leonurine. The lab will design relative experiments to further illustrate this issue.

In conclusion, Leonurine induced cardiac protection effects in vivo in MI by activating the PI3K/AKT/GSK3 $\beta$ signaling pathway, which promoted the expression of anti-apoptosis proteins, decreased the expression of pro-apoptosis proteins and inhibits the activity of cleaved-caspase 3 . It indicates that Leonurine represents a promising drug in treating ischemic heart diseases.

\section{Acknowledgements}

Not applicable.

\section{Funding}

The experimental performance is supported by National Nature Science Foundation of China; contract grant no. 81100130; Specialized Research Fund for the Doctoral Program of Higher Education; contract grant no. 20120141110013; National Key Basic Research Program of China; contract grant no. 81370283; 2005CB623903.

\section{Availability of data and materials}

The datasets used and/or analyzed during the current study are available from the corresponding author on reasonable request.

\section{Authors' contributions}

LX, HLZ and XJJ designed the experiments. HLZ and FW performed the experiments. LX and HLZ analyzed the data. LX, HLZ and XJJ wrote the manuscript. All authors read and approved the final manuscript.

\section{Ethics approval and consent to participate}

The study was approved by the Ethics Committee of Renmin Hospital of Wuhan University.

\section{Consent for publication}

Not applicable. 


\section{Competing interests}

The authors declare that they have no competing interests.

\section{References}

1. Zhu YZ, Chong CL, Chuah SC, Huang SH, Nai HS, Tong HT, Whiteman $\mathrm{M}$ and Moore PK: Cardioprotective effects of nitroparacetamol and paracetamol in acute phase of myocardial infarction in experimental rats. Am J Physiol Heart Circ Physiol 290: H517-H524, 2006.

2. Liu C, Guo W, Maerz S, Gu X and Zhu Y: 3,5-Dimethoxy4-(3-(2-carbonyl-ethyldisulfanyl)-propionyl)-benzoic acid 4-guanidino-butyl ester: A novel twin drug that prevents primary cardiac myocytes from hypoxia-induced apoptosis. Eur J Pharmacol 700: 118-126, 2013.

3. Gallogly MM, Shelton MD, Qanungo S, Pai HV, Starke DW, Hoppel CL, Lesnefsky EJ and Mieyal JJ: Glutaredoxin regulates apoptosis in cardiomyocytes via NFkappaB targets Bcl-2 and Bcl-xL: Implications for cardiac aging. Antioxid Redox Signal 12: 1339-1353, 2010

4. Lee Y and Gustafsson AB: Role of apoptosis in cardiovascular disease. Apoptosis 14: 536-548, 2009.

5. Haunstetter A and Izumo S: Toward antiapoptosis as a new treatment modality. Circ Res 86: 371-376, 2000

6. Liu XH, Xin H and Zhu YZ: More than a 'mother-benefiting' herb: Cardioprotective effect of Herba leonuri. Sheng Li Xue Bao 59: 578-584, 2007.

7. Kuang PG, Zhou XF, Zhang FY and Lang SY: Motherwort and cerebral ischemia. J Tradit Chin Med 8: 37-40, 1988.

8. Wang ZS, Li DW, Xia WJ, Qiu HQ and Zhu LY: The therapeutic effect of herba leonuri in the treatment of coronary myocardial ischemia. J Tradit Chin Med 8: 103-106, 1988.

9. Liu XH, Xin H, Hou AJ and Zhu YZ: Protective effects of leonurine in neonatal rat hypoxic cardiomyocytes and rat infarcted heart. Clin Exp Pharmacol Physiol 36: 696-703, 2009.

10. Liu XH, Chen PF, Pan LL, Silva RD and Zhu YZ: 4-Guanidino-n-butyl syringate (Leonurine, SCM 198) protects $\mathrm{H} 9 \mathrm{c} 2$ rat ventricular cells from hypoxia-induced apoptosis. J Cardiovasc Pharmacol 54: 437-444, 2009.

11. Liu X, Pan L, Gong Q and Zhu Y: Leonurine (SCM-198) improves cardiac recovery in rat during chronic infarction. Eur J Pharmacol 649: 236-241, 2010.

12. Liu XH, Pan LL, Deng HY, Xiong QH, Wu D, Huang GY, Gong QH and Zhu YZ: Leonurine (SCM-198) attenuates myocardial fibrotic response via inhibition of NADPH oxidase 4. Free Radic Biol Med 54: 93-1042, 2013.

13. Liu H, Zhang X, Du Y, Ji H, Li S, Li L, Xing Y, Zhang X, Dong L, Wang $\mathrm{C}$, et al: Leonurine protects brain injury by increased activities of UCP4, SOD, CAT and Bcl-2, decreased levels of MDA and Bax, and ameliorated ultrastructure of mitochondria in experimental stroke. Brain Res 1474: 73-81, 2012.

14. Yu-Shengyou and LI Y: Dexamethasone inhibits podocyte apoptosis by stabilizing the PI3K/Akt signal pathway. Biomed Res Int 2013: 326986, 2013

15. Franke TF, Hornik CP, Segev L, Shostak GA and Sugimoto C: PI3K/Akt and apoptosis: size matters. Oncogene 22: 8983-8998, 2003.

16. Fresno Vara JA, Casado E, De Castro J, Cejas P, Belda-Iniesta C and González-Barón M: PI3K/Akt signalling pathway and cancer. Cancer Treat Rev 30: 193-204, 2004.
17. Ohashi H, Takagi H, Oh H, Suzuma K, Suzuma I, Miyamoto N, Uemura A, Watanabe D, Murakami T, Sugaya T, et al: Phosphatidylinositol 3-kinase/Akt regulates angiotensin II-induced inhibition of apoptosis in microvascular endothelial cells by governing survivin expression and suppression of caspase-3 activity. Circ Res 94: 785-793, 2004.

18. Tramontano AF, Muniyappar R, Black AD, Blendea MC, Cohen I, Deng L, Sowers JR, Cutaia MV and El-Sherif N: Erythropoietin protects cardiac myocytes from hypoxia-induced apoptosis through an Akt-dependent pathway. Biochem Biophys Res Commun 308: 990-994, 2003.

19. Cai $\mathrm{Z}$ and Semenza GL: Phosphatidylinositol-3-kinase signaling is required for erythropoietin-mediated acute protection against myocardial ischemia/reperfusion injury. Circulation 109: 2050-2053, 2004.

20. Rayasam GV, Tulasi VK, Sodhi R, Davis JA and Ray A: Glycogen synthase kinase 3: More than a namesake. Br J Pharmacol 156: 885-98, 2009

21. Maixner DW and Weng HR: The role of glycogen synthase kinase 3 beta in neuroinflammation and pain. J Pharm Pharmacol (Los Angel) 1: 001, 2013.

22. Cross DA, Alessi DR, Cohen P, Andjelkovich M and Hemmings BA: Inhibition of glycogen synthase kinase- 3 by insulin mediated by protein kinase B. Nature 378: 785-789, 1995.

23. Descamps S, Pawlowski V, Révillion F, Hornez L, Hebbar M, Boilly B, Hondermarck H and Peyrat JP: Expression of nerve growth factor receptors and their prognostic value in human breast cancer. Cancer Res 61: 4337-4340, 2001.

24. Morisco C, Zebrowski D, Condorelli G, Tsichlis P, Vatner SF and Sadoshima J: The Akt-glycogen synthase kinase 3beta pathway regulates transcription of atrial natriuretic factor induced by beta-adrenergic receptor stimulation in cardiac myocytes. J Biol Chem 275: 14466-14475, 2000.

25. Badorff C, Ruetten H, Mueller S, Stahmer M, Gehring D, Jung F, Ihling C,Zeiher AM and Dimmeler S: Fas receptor signaling inhibits glycogen synthase kinase 3 beta and induces cardiac hypertrophy following pressure overload. J Clin Invest 109: 373-381, 2002.

26. Gong R, Rifai A and Dworkin LD: Activation of PI3K-Akt-GSK3beta pathway mediates hepatocyte growth factor inhibition of RANTES expression in renal tubular epithelial cells. Biochem Biophys Res Commun 330: 27-33, 2005.

27. Wagner C, Tillack D, Simonis G, Strasser RH and Weinbrenner C: Ischemic post-conditioning reduces infarct size of the in vivo rat heart: Role of PI3-K, mTOR, GSK-3beta and apoptosis. Mol Cell Biochem 339: 135-147, 2010.

28. Somervaille TC, Linch DC and Khwaja A: Growth factor withdrawal from primary human erythroid progenitors induces apoptosis through a pathway involving glycogen synthase kinase-3 and Bax. Blood 98: 1374-1381, 2001

29. Sun J, Huang SH, Zhu YC, Whiteman M, Wang MJ, Tan BK and Zhu YZ: Anti-oxidative stress effects of Herba leonuri on ischemic rat hearts. Life Sci 76: 3043-3056, 2005.

30. Liu X, Pan L, Wang X, Gong Q and Zhu YZ: Leonurine protects against tumor necrosis factor- $\alpha$-mediated inflammation in human umbilical vein endothelial cells. Atherosclerosis 222: 34-42, 2012.

31. Loh KP, Qi J, Tan BK, Liu XH, Wei BG and Zhu YZ: Leonurine protects middle cerebral artery occluded rats through antioxidant effect and regulation of mitochondrial function. Stroke 41: 2661-2668, 2010.

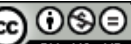

This work is licensed under a Creative Commons Attribution-NonCommercial-NoDerivatives 4.0 International (CC BY-NC-ND 4.0) License. 